EPJ Web of Conferences 41, 02019 (2013)

DOI: $10.1051 /$ epjconf/20134102019

(C) Owned by the authors, published by EDP Sciences, 2013

\title{
When does an electron exit a tunneling barrier?
}

\author{
D. Shafir ${ }^{1}$, H. Soifer ${ }^{1}$, B. D. Bruner ${ }^{1}$, M. Dagan ${ }^{1}$, Y. Mairesse ${ }^{2}$, S. Patchkovskii ${ }^{3}$, M. Yu. Ivanov ${ }^{4,5}$, \\ O. Smirnova ${ }^{5}$, and N. Dudovich ${ }^{1}$
}

${ }^{1}$ Department of Physics of Complex Systems, Weizmann Institute of Science, Rehovot 76100, Israel

${ }^{2}$ CELIA, Universite Bordeaux I, UMR 5107 (CNRS, Bordeaux 1, CEA), 351 Cours de la Liberation, 33405 Talence Cedex, France

${ }^{3}$ National Research Council of Canada, 100 Sussex Dr., Ottawa, Ontario K1A 0R6, Canada

${ }^{4}$ Department of Physics, Imperial College London, South Kensington Campus, SW7 2AZ London, United Kingdom

${ }^{5}$ Max-Born Institute for Nonlinear Optics and Short Pulse Spectroscopy, Max-Born-Strasse 2A, D12489 Berlin, Germany

\begin{abstract}
We probe the dynamics of tunnel ionization via high harmonic generation. We characterize the ionization dynamics in helium atoms, and apply our approach to resolve subtle differences in ionization from different orbitals of a $\mathrm{CO}_{2}$ molecule.
\end{abstract}

Tunneling of a particle through a barrier is one of the most fundamental and ubiquitous quantum processes. When induced by an intense laser field [1], electron tunneling from atoms and molecules initiates a broad range of processes that evolve on an attosecond time-scale. As the liberated electron is driven by the laser field, it can return to the parent ion and recombine to the initial (ground) state, consequently releasing its energy in an attosecond burst of light [2]. This process, known as High Harmonic Generation (HHG), serves as an excellent spatio-temporal filter for the electron motion.

In our recent work we have demonstrated how the time at which the electron leaves the atomic barrier can be directly measured [3]. Specifically, we applied a strong laser field to induce tunneling and a weak probe field to steer the tunneled electron. By monitoring attosecond pulses emitted when the liberated electron re-encounters the parent ion, both ionization times and recollision times were reconstructed. We demonstrated high sensitivity of the measurement by resolving subtle delays in ionization times from two orbitals of a $\mathrm{CO} 2$ molecule.

To characterize ionization and recombination times independently, we need a probe that is both perturbative and sufficiently fast to monitor these electron trajectories on a sub-cycle time scale [4]. Such a probe is introduced by adding a weak second harmonic $(\mathrm{SH})$ field, orthogonally polarized to the strong fundamental beam. When the weak field interacts with the free electron it induces a lateral displacement, leading to a reduced recollision probability. The second harmonic field acts as a sub-cycle gate, selecting a narrow range of trajectories that return to the parent ion. As the two-color delay is changed, the gate is shifted within the cycle, and consequently a different ionization window is chosen. An additional gate is induced by the lateral velocity. This velocity as well changes within the optical cycle and depends on both the ionization times and recombination times. In spherically symmetric systems we can extract this velocity by calculating the ratio between even and odd harmonics [5]. This ratio maximizes when the lateral velocity is maximal. The ratio of even to odd

This is an Open Access article distributed under the terms of the Creative Commons Attribution License 2.0, which permits unrestricted use, distribution, and reproduction in any medium, provided the original work is properly cited. 
harmonic amplitudes thus provides an additional independent observable that allows us to disentangle the ionization and recombination times.

We first generate high harmonics from helium atoms using a strong $\lambda=800 \mathrm{~nm}$ fundamental field and a weak second harmonic field at a $1 \%$ intensity level. We measure the harmonic intensity as a function of the two-color delay $\phi$. This measurement reflects the 'displacement gate'. Figure 1A presents the normalized harmonic intensities versus the two-color delay, at a field intensity of $3.8 \times 10^{14} \mathrm{~W} / \mathrm{cm}^{2}$. The delay for which the signal is maximal, $\phi_{\text {max }}^{\mathrm{d}}$, changes with harmonic order, reflecting its dependence on the trajectory. In addition, we measure the ratio between adjacent even and odd harmonics, which reflects the 'velocity gate' (Fig. 1B). Again, we extract the delay $\phi^{\mathrm{v}}{ }_{\max }$, where this ratio is maximized by the 'velocity gate'. The $\phi$-dependence for the two observables is significantly different, reflecting their independence.
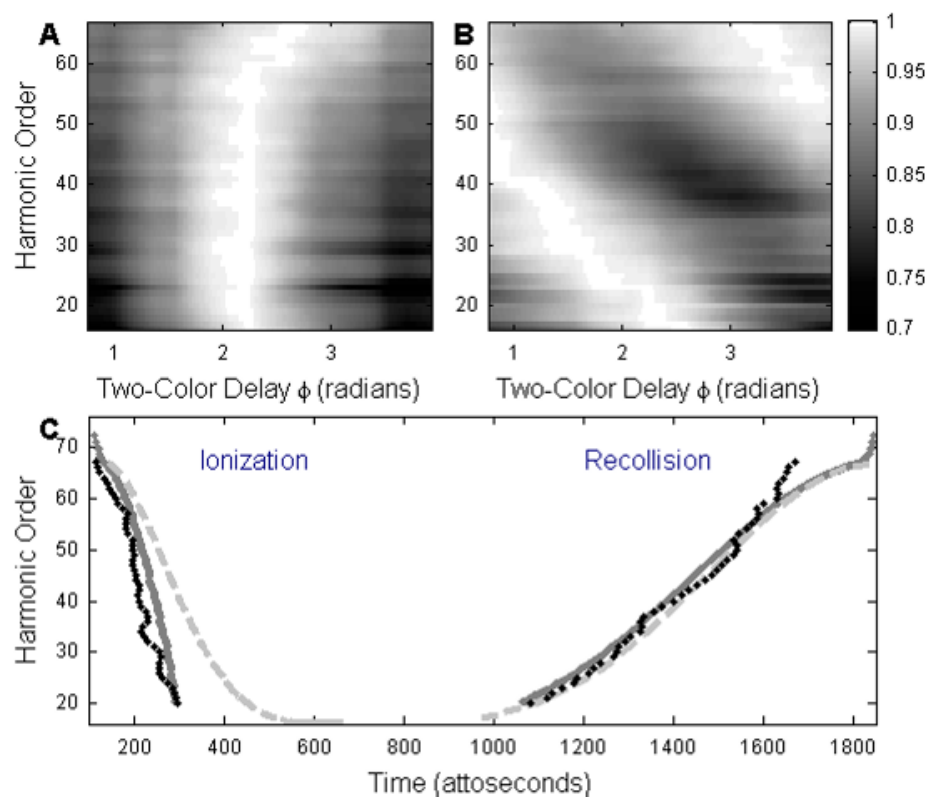
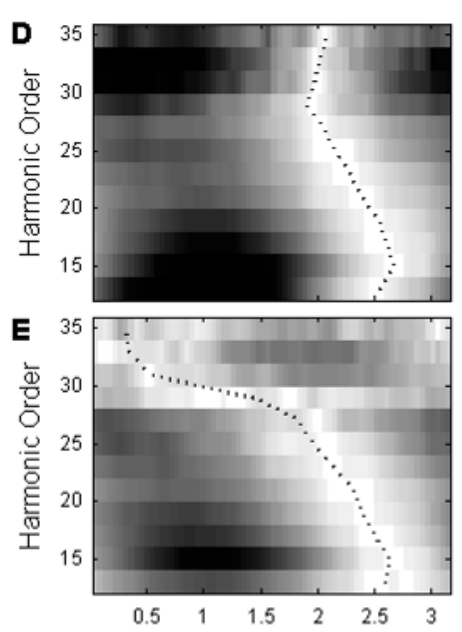

Two-Color Delay $\phi$ (radians)

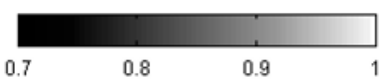

Fig. 1. A. Displacement gate: normalized harmonic signal as a function of $\phi$. B. Velocity gate: recollision angle as a function of $\phi$. C. Reconstructed ionization and recollision times extracted from A and B. The extracted times (black dots) are compared to the calculated times according to the classical model (light dashed curves) and the quantum stationary solution (dark solid curves). D-E. Displacement gate for $\mathrm{CO}_{2}$ molecules aligned at 90 (D) and $0(E)$ degrees relative to the probe polarization. Black dotted lines mark $\phi^{\mathrm{d}}$ max.

The perturbative nature of the gate allows us to reconstruct both the ionization time and the recollision time. We calculate both the lateral shift and lateral velocity of the recolliding electron assuming that both times are dictated by the strong fundamental field. Figure $1 \mathrm{C}$ presents the reconstructed ionization and recombination times (black dots) together with the predictions of the standard semi-classical model [2] (light dashed curves). The reconstructed recombination times agree well with the semi-classical model. As for the ionization times, a distinct deviation is observed for the lower harmonics: the reconstructed ionization window is about 100 attoseconds shorter than the semi-classical one. This deviation is explained by the stationary solution [6]. When an electron, bound by the ionization potential, enters at some moment $t_{0}$ the tunneling barrier induced by the strong laser field, its kinetic energy becomes negative [6]. The stationary solution in this case for the initial velocity and initial time is complex. The quantum exit (ionization) time, $t_{i}$, is defined as the real part of the complex-valued ionization time. Under our experimental conditions, it differs from 
the semi-classical exit time by up to a few hundred attoseconds, as shown by the dark solid line in Fig. 1C.

The next step is the probing of molecular systems where more than one ionization channel can participate in the process [7]. Specifically, we focus on aligned $\mathrm{CO}_{2}$ molecules where both the HOMO and the HOMO-2 contribute to the tunneling process. Figures 1D, E present the normalized harmonic intensity as a function of the two-color delay $\phi$ for $\mathrm{CO}_{2}$ molecules aligned at 90 and 0 degrees correspondingly. For perpendicular alignment (panel D), a single orbital (HOMO) dominates the signal, therefore the maximum of the signal $\phi^{\mathrm{d}}{ }_{\max }$ changes smoothly with the harmonic order. As we rotate the molecule to be parallel to the laser field, we switch on the contribution of the HOMO-2 (panel E) [7]. In this case we observe a dramatic shift of $\phi_{\text {max }}^{d}$ by $\sim \pi / 2$ around harmonic 29 . This harmonic order corresponds to a minimum observed in the signal, attributed to a destructive interference between the two orbitals [7]. This dramatic shift reveals that two orbitals contribute to the process, resolving subtle delays in their ionization times [3].

In most molecular systems ionization involves attosecond core rearrangements that may lead to a real time-delay associated with non-trivial phases between different tunneling channels. Timeresolving the tunneling dynamics in complex molecular systems will provide a deep insight into fundamental multielectron phenomena -- the long-term goal of attosecond science.

\section{References}

1. L. V. Keldysh, JETP 20, 1307-1314 (1965).

2. P. Corkum, PRL 71, 1994-1997 (1993).

3. D. Shafir, H. Soifer, B. D. Bruner, M. Dagan, Y. Mairesse, S. Patchkovskii, M. Y. Ivanov, O. Smirnova, and N. Dudovich, Nature 485, 343-346 (2012).

4. N. Dudovich, O. Smirnova, J. Levesque, Y. Mairesse, M. Y. Ivanov, D. Villeneuve, and P. B. Corkum, Nat. Phys. 2, 781-786 (2006).

5. D. Shafir, Y. Mairesse, D. Villeneuve, P. B. Corkum, and N. Dudovich, Nat. Phys. 5, 412-416 (2009).

6. M. Lewenstein, P. Balcou, M. Y. Ivanov, A. L'Huillier, and P. B. Corkum, PRA 49, 2117 (1994).

7. O. Smirnova, Y. Mairesse, S. Patchkovskii, N. Dudovich, D. Villeneuve, P. B. Corkum, and M. Y. Ivanov, Nature 460, 972-7 (2009). 